\title{
La representación mediática de la violencia escolar: El caso de Avellaneda (Argentina)
}

C Copyright 2021. Universidad Nacional Autónoma de Nicaragua, Managua (UNAN-Managua) Todos los derechos reservados

\section{The media representation of school violence: The case of Avellaneda (Argentina)}

Luis Héctor Amaranto Barreras

Universidad Nacional de Avellaneda

Universidad Nacional de La Plata

https://orcid.org/0000-0002-0513-4820

lbarreras@undav.edu.ar

\section{Ayelén Suyay Gómez}

Universidad Nacional de Avellaneda

https://orcid.org/0000-0003-4531-8912

agomez@undav.edu.ar

Fecha de recibido: 10/04/2021

\section{Resumen}

El presente trabajo forma parte del Proyecto de Investigación UNDAVCYT: "La representación mediática de la Violencia Escolar: Lo audiovisual como herramienta de intervención.", subsidiado en su convocatoria 2017 y ejecutado en el transcurso de los últimos años, el cual se desarrolló desde el Departamento de Ciencias Sociales de la Universidad Nacional de Avellaneda.

El eje central es el de promover espacios de aprendizaje en el sistema educativo, mediante la producción de materiales audiovisuales, que brinden las herramientas necesarias para reflexionar acerca de las causas y las nociones de la violencia escolar. En esa línea, debemos preguntarnos, ¿qué se señala como violencia en las escuelas?, ¿qué universo construyen los medios de comunicación sobre la violencia y las causas de ella? ¿Qué visión tienen los jóvenes sobre

\section{Milena Hidalgo}

Universidad Nacional de Avellaneda

https://orcid.org/0000-0001-5290-9580

mhidalgo@undav.edu.ar

\section{Camila Szulz}

Universidad Nacional de Avellaneda

https://orcid.org/0000-0002-8663-1270

camilaszulzo9@gmail.com

Fecha de dictaminado: 30/04/2021 el tema? entre otras. La importancia de este proyecto radica en promover la intervención en el sistema educativo, a través de la herramienta audiovisual como lugar de ruptura y creación donde más jóvenes puedan intervenir en los procesos de producción de conocimiento sobre la denominada "violencia escolar".

En definitiva, la propuesta promueve un diálogo articulado entre los análisis de las representaciones mediáticas, las nociones centrales de este debate y la voz de los/as estudiantes para desarrollar productos audiovisuales que contengan la mirada de los jóvenes sobre la problemática de la violencia en ámbitos educativos en la localidad de Avellaneda (Argentina).

\section{Palabras claves}

Escuela, medios, jóvenes y violencia. 


\section{Summary}

This work is part of the UNDAVCYT Research Project: "The media representation of School Violence: The audiovisual as an intervention tool.", Subsidized in its 2017 call and executed in the course of recent years, which was developed from the Department of Social Sciences of the National University of Avellaneda.

The central axis is to promote learning spaces in the educational system, through the production of audiovisual materials, which provide the necessary tools to reflect on the causes and notions of school violence. Along these lines, we must ask ourselves, what is designated as violence in schools? What universe does the media build on violence and its causes? What vision do young people have on the subject? among other. The importance of this project lies in promoting intervention in the educational system, through the audiovisual tool as a place of rupture and creation where more and more young people can intervene in the processes of knowledge production about the so-called "school violence".

In short, the proposal promotes an articulated dialogue between the analysis of media representations, the central notions of this debate and the voice of students to develop audiovisual products that contain the gaze of young people on the problem of violence in educational settings, in the town of Avellaneda (Argentina).

\section{Keywords}

School, media, young boys and violence.

\section{Introducción}

En la contemporaneidad vemos un relato mediático dominante, a través de mecanismos de simplificación, descontextualización y deshistorización, y lo primero que aparece es la idea de etiquetar a un sector de la juventud en construcciones de diversos acontecimientos como el caso de Carmen de Patagones, donde aparece la victimización del sujeto o la criminalización del mismo, la voz de autoridad y raramente se escucha la opinión de los/as jóvenes. En esa línea, el estudio del proyecto parte de la referencia de los estudios sobre las juventudes analizando a las mismas desde una perspectiva de derechos y no de consumo como lo hace el mercado.
El estudio de las relaciones entre la realidad y la actuación mediática nos permitirá acceder a un territorio social, influido por el reconocimiento de nuevos modos de participación. Los cambios representacionales en las producciones audiovisuales habilitan a suponer que este objeto de estudio que se encuentra en pleno desarrollo, estimulará el control crítico, la racionalización de políticas específicas, una mayor comprensión operativa para la planificación y gestión de estrategias y políticas culturales para prevenir la violencia.

Esta iniciativa contempló lo audiovisual como herramienta de intervención desde la producción de materiales audiovisuales, con fines pedagógicos para colegios secundarios públicos de la localidad de Avellaneda (Colegio Padre Berisso de la localidad de Avellaneda y el Colegio Secundario el (astillito). Promoviendo la vinculación entre extensión, docencia e investigación a través de la realización de productos audiovisuales (material de investigación y análisis), así como el seguimiento por parte de los integrantes del proyecto, en los procesos de elaboración y comunicación del producto con los establecimientos educativos a trabajar.

El proyecto de investigación "La representación mediática de la Violencia escolar: Lo audiovisual como herramienta de intervención" contempló la problemática a partir de la mal llamada "violencia en las escuelas" de diversos casos. A la hora de reflexionar sobre esta temática, también hay que observar a los jóvenes, a la representación mediática sobre la juventud, sus pensamientos, sus acciones, sus miradas y representaciones. Los medios de comunicación nos ponen en contacto casi permanente con la violencia que existe en nuestra sociedad y la que se crea de forma imaginaria. Probablemente, por eso son considerados con frecuencia como una de las principales causas que origina la violencia en los niños y en los jóvenes.

En torno a este tema el proyecto permitió pensar la posibilidad y conveniencia de utilizar la tecnología audiovisual con carácter educativo, para prevenir estos fenómenos. En la actualidad, las personas configuran gran parte de su identidad a través de los medios de comunicación; en ese sentido, un modelo de prevención de la violencia tiene que enseñarnos a "leer" y a descifrar lo que construyen los medios. De este modo, el relevamiento previsto apuntó a ver de qué manera los medios construyeron la realidad sobre la violencia escolar, bajo qué parámetros definieron estos sucesos y como identificaban a los y las jóvenes. 
La investigación que se basó hasta el presente en la contextualización histórica por medio de antecedentes y paradigmas previos a la aparición de la "Violencia Escolar", así como en la observación analítica y crítica de sus efectos y de sus consecuencias en los jóvenes, nos impulsa a obtener una ampliación del sentido en esta perspectiva.

permite dar paso a la presentación y resultados del estudio que ha dado origen a este texto.

\section{Los Objetivos}

Desde el armado del proyecto se buscó como objetivo central, promover espacios de aprendizaje en el sistema educativo mediante la producción de materiales audiovisuales, que brinden las herramientas necesarias para reflexionar acerca de las causas y las nociones de la violencia escolar. Entre los ejes específicos está el de promover la voz de los/as estudiantes para desarrollar productos audiovisuales sobre la problemática de la violencia en ámbitos educativos. En esa línea, es importante reconocer el valor de la imagen como fuente de conocimiento, de lenguaje y como herramienta para propiciar la intervención en el ámbito escolar.

Por un lado, las charlas que se desarrollaron con los/as estudiantes y docentes de los colegios Padre Berisso de la localidad de Avellaneda y el Colegio Secundario el Castillito, fueron grabadas y de cada una de ellas se generó una memoria audiovisual que contiene el diálogo virtual que se desarrolló con dichos colegios. Por otro lado, se realizó un pequeño corto con voces de docentes de colegios secundarios, profesionales de la Universidad Nacional de Avellaneda que trabajan en vinculación con los colegios secundarios. Dicho material también fue generado de forma virtual y cada entrevista fue grabada por los propios informantes con una consigna que le habíamos enviado. Este corto audiovisual funciona como herramienta pedagógica en el ámbito educativo, para promover debates y reflexiones en torno a las nociones que circulan en el imaginario colectivo sobre la mal llamada "Violencia Escolar".

\section{Una aproximación al marco teórico- conceptual: Los Jóvenes}

La palabra "joven" implica una categoría social para definir a un sector de la población. La misma, es producto de una cultura y como tal constituye una concepción contingente, es decir que va variando sus características de acuerdo al contexto histórico y social. Para posicionar el surgimiento de esta noción, muchos autores la sitúan en el fin de la primera guerra mundial y sus años posteriores, sobre todo en la reafirmación de ciertos movimientos juveniles de principios del siglo XX en Alemania e Inglaterra. Pero especialmente, Rossana Reguillo Cruz (2005, p. 6) es quien plantea un debate interesante en esta irrupción: "La juventud como hoy la conocemos es propiamente una invención de la posguerra, en el sentido del surgimiento de un nuevo orden internacional que conformaba una geografía política en la que los vencedores accedían a inéditos estándares de vida e imponían sus estilos y valores. La sociedad reivindicó la existencia de los niños y los jóvenes como sujetos de derechos y, especialmente, en el caso de los jóvenes como sujetos de consumo"

\section{Violencias}

“¿Qué mayor evidencia de que algo no marcha bien en nuestras sociedades y del malestar profundo que nos habita pensar en el caso de Junior? Él no encontró en la sociedad a la que pertenece, razones suficientes para valorar la vida de los otros, la suya propia y sentirse responsable. Contemplar la muerte de los otros jóvenes es mirar de frente el espejo que devuelve lo que se pretende eludir: la responsabilidad social, cuya disolución hace posible que tanto en Litletton (la Columbine de Michael Moore), como en Inglaterra, como en la Argentina y México aparezcan jóvenes y niños asesinados" (Reguillo Cruz, 2005 s/p).

En estos últimos años, las noticias de los jóvenes, de la escuela, se han trasladado del "género" educativo al policial. En las aulas argentinas y fuera de ellas, se registraron acontecimientos violentos protagonizadas por estudiantes armados, disputas entre jóvenes, discriminación por parte de compañeros o profesores y hechos graves de docentes agredidos por los propios estudiantes o por los padres de los y las adolescentes.

Cuando se habla de "Violencia Escolar", deberíamos preguntarnos si ocurre en la escuela, qué características disímiles posee de la que se da en otros espacios; es decir, el planteo que etiquetan los medios de comunicación y la sociedad como un paquete de la "violencia escolar" producto de la escuela, que se circunscribe a los límites geográficos de la institución y se analiza de forma banal, desde el sentido común. La primera estrategia que proponemos para pensar este debate, sería no enunciar estos hechos como 
"Violencia Escolar", o por lo menos usar las comillas, para poder entender que la escuela y los/as jóvenes que son parte de estos acontecimientos, no están más allá de lo social y esos hechos son parte de una trama compleja que debe ser tratada con racionalidad, serenidad, evitando la sentencia social.

\section{La representación mediática de la "violencia juvenil"}

Respecto de la construcción mediática de los y las jóvenes como un enemigo, la investigadora mexicana Rossana Reguillo (1997) dice:

"Mientras que en un recuadro aparece la opinión de un especialista en niños de la calle, pero esta aparece sin problematización alguna y sus opiniones, por el tratamiento que de ellas se hace, lejos de cuestionar las visiones oficiales o de confrontarlas, las confirma y el asunto se coloca en términos de comprensión para los pobres delincuentes juveniles. Se pasa así de la estigmatización al sentimiento caritativo, lo cual no permite trascender la percepción simplista que reduce la complejidad del fenómeno aludido a un asunto entre buenos y malos"

De esta forma, se debería analizar los casos desde los contextos, representando sus realidades sociales, culturales, económicas, etc. No tomándolos como temas aislados o estancos sino como vínculos simbólicos que nos permitirán obtener un diagnóstico para poder planificar políticas públicas sobre dicha problemática. La violencia es el tema, pero lo que está en juego es el peso social que están cobrando las imágenes que este país se hace de sí mismo cotidianamente en la radio y la televisión, además de las contradictorias concepciones de la comunicación que mediatizan lo que creíamos saber acerca de los medios. Para comprender esta relación de las violencias y las pantallas se debe tener en cuenta el momento de privatización de la vida y la disolución del espacio público donde se pierde el sentido de la calle como ámbito de comunicación y reconocimiento.

En la contemporaneidad, vemos un relato dominante desde los medios masivos de comunicación a través de mecanismos de simplificación, descontextualización y deshistorización, y lo primero que aparece es la idea de etiquetar a un sector de la juventud porque es simple para decodificar, para interpretar una idea y para obturar un sentido. Eso construye procesos de estigmatización de los y las jóvenes. Cuando se habla de "violencia escolar" no se discute sobre la violencia de las normas por cuestiones de género, etnia, diversidad, violencia de los adultos en los jóvenes. Esta idea planteada desde la cobertura de los acontecimientos, implica el reduccionismo al buscar como únicas causas de la violencia: la droga, el alcohol, la televisión, los videojuegos o las nuevas tecnologías.

Las violencias se diversifican, alimentándose a símismas del miedo, de la incertidumbre, de la desesperanza y de la disolución del vínculo social. Desde esta perspectiva, el texto pretende ser un ensayo crítico que permita comprender cuál es la relación existente entre los/as jóvenes, la violencia y los medios de comunicación, señalando la importancia de los hechos como vínculos simbólicos desde los contextos, representando sus realidades sociales, culturales, políticas, económicas e históricas.

Ante el actual panorama de transformaciones, los/ as jóvenes han sido afectados en su percepción de la política, del espacio y del futuro, que "es en el campo de las expresiones culturales donde los jóvenes se vuelven visibles como actores sociales" (Reguillo Cruz, 2000, s/p). En torno a este tema el proyecto permite pensar la posibilidad y conveniencia de utilizar la tecnología audiovisual con carácter educativo, para prevenir estos fenómenos. En la actualidad, las personas disponen gran parte de su identidad a través de los medios de comunicación, por lo tanto es necesario un modelo de prevención de la violencia que nos enseñe a analizar y descifrar lo que construyen los medios.

Pensar en la violencia aislada de la revisión de la historicidad de la Argentina, su construcción política, económica y cultural no permitiría comprender la complejidad de los relatos mediáticos de la violencia en los y las jóvenes en la actualidad. A partir de la identificación del concepto de violencia en la comunidad educativa, es como se puede trabajar en la prevención y de esta manera, lograr la transformación de dichos acontecimientos.

\section{Metodología}

La presente investigación se apoyó en la metodología cualitativa, la cual permite analizar los procesos y fenómenos sociales, prácticas, instituciones y patrones de comportamiento, para desentrañar los significados construidos alrededor de ellos, en un contexto o 
entorno que puede ser de redes de relaciones sociales, sistemas de creencias, rituales, etc. Justamente este contexto es el que permite la tarea interpretativa (Sautu, 2003).

Por otra parte, la metodología cualitativa es "apropiada cuando el investigador se propone investigar la construcción social de significados, las perspectivas de los actores sociales, las condiciones de la vida cotidiana o brindar una descripción detallada de la realidad" (Weinerman, C y Sautu, R, 1997).

El trabajo de campo se planteó en dos etapas y con dos formas de cumplimiento:

En una primera etapa, realizamos tareas de carácter exploratoria y descriptiva, con seguimientos amplios, de aproximación y detección de informantes principales y contextuales, pero con recolección de información asistemática (organización y análisis multimedia de antecedentes, fuentes documentales, archivos, mediáticas, publicaciones especializadas, teorías.). En este trabajo se utilizará la observación participante con la inclusión de entrevistas estructuradas, y también informales. Como se mencionó, se desarrollaron recursos técnicos propios de las investigaciones cualitativas. De manera paralela, se llevó adelante una estrategia metodológica cuantitativa que nos permitió sistematizar los datos y recabar información.

En cuanto a la perspectiva metodológica utilizada, se hizo propia la perspectiva:

Bajo la premisa de que la diversidad sociocultural del mundo supone una diversidad epistemológica, es decir una ecología de saberes. Para ello, es fundamental generalizar las prácticas integrales en los y las estudiantes para la convivencia natural de la investigación y extensión en el cursado, también en la incorporación desde un vínculo dialógico-crítico con actores sociales organizados y en procesos de luchas (Medina, Tommasino, 2018).

Se trabajó sobre la integralidad de las funciones sustantivas de la universidad y la incorporación de metodología de la denominada "Extensión Crítica", mapeo de actores y territorios, los cuales permiten identificar actores, situaciones de poder, y relaciones. De esta manera, pensamos el proceso de investigación como parte estructurada y en dialéctica relación con la extensión desde la integralidad de las funciones sustantivas de la universidad.
La investigación- acción participativa y la extensión crítica vinculada a la idea expuesta de integralidad de las funciones, posibilitó diseñar un proyecto para ampliar nuestros objetivos e incorporar demandas y necesidades de los colectivos laborales. Propusimos una metodología participativa que se realizó en el campo. Mediante las técnicas utilizadas (entrevistas individuales o grupales, observación participante, talleres en los espacios de trabajo) donde se detectaron variables que intervienen en la situación problemática. Por ellos diseñamos como estrategia metodológica una estructura socio- organizacional- comunicacional, donde se determina el trabajo, los vínculos y las comunicaciones, para la resolución de conflictos.

\section{Resultado y discusiones}

Como estructura de trabajo se realizaron análisis de la representación del imaginario juvenil de los diarios de Avellaneda en torno al estudio de la violencia escolar y por otro lado se promovió la voz de los/as estudiantes para entender dicha problemática y coproducir conocimientos a través del diálogo de saberes. En esta línea compartimos fragmentos de los resultados del análisis y las discusiones realizadas.

\section{Análisis de la comunicación de la violencia escolar}

En primera instancia, antes de pensar en posibles proyectos para colaborar con el fin del acoso escolar, deliberamos la necesidad de tener un panorama de la situación actual de esta problemática. Para ello, analizamos los modos de la representación del imaginario juvenil de los diarios de Avellaneda en torno al estudio de la violencia escolar. Las unidades de observación fueron notas periodísticas de diversos diarios digitales y portales de Internet (de la localidad de Avellaneda, Wilde y Lanús). En este caso, todos los hechos con los que trabajamos ocurrieron en la zona sur de la Provincia de Buenos Aires, lugar donde en primera medida llevaremos a cabo nuestro proyecto.

El 20 de agosto de 2013 el Diario Digital Popular titula: "Más polémica entre la Técnica 5 de Avellaneda y del Normal Próspero Alemandri". En la nota, se informa cómo padres y alumnos siguen la disputa sobre cuál de los dos establecimientos educativos tiene más "aguante" en las redes sociales. En sus líneas refiere que previamente, hubo dos ataques por parte de los jóvenes. Los adultos, lejos erradicar los hechos de violencia, siguieron la discusión de manera virtual. 
“Aguante el Palaá", "aguante nada, anda a tirar piedras a la cancha", fue una de las frases que se puedo leer. Por otra parte, Sabrina, madre de un alumno del ENSPA aseguró: "sufrí en carne propia la agresión de esos delincuentes en la puerta del jardín de infantes. Si no van a ir presos por los destrozos, que los padres de ellos paguen los arreglos. Por suerte ningún niño resultó herido". En cuanto a las autoridades de ambas instituciones, también realizaron su descargo de manera digital, aunque tampoco ayudaron a apaciguar el conflicto.

En la página del ENSPA se posteó el lema "la educación es la semilla que germina y crece, enriqueciendo al hombre". Desde la técnica 5, una fuente aseguró que "los del Normal pasaron por acá otra vez y con algunos padres amenazando, los alumnos querían salir, el director se opuso y hasta hubo forcejeos en la escuela. Hay que parar todo esto ya".

El 21 de octubre del mismo año, el diario Clarín menciona denuncias por bullying en la secundaria $\mathrm{N}^{\circ} 23$ de Wilde, Avellaneda, a raíz de un video sobre la agresión de Agustín Romero, un adolescente de 15 años de dicho establecimiento. En la grabación se puede ver como sus compañeros lo empujan y le dan trompadas mientras que otros se ríen a su alrededor. Desde Educación de la Provincia informaron que "van a investigar lo que sucedió y los grados de responsabilidades que corresponden a adultos y alumnos y en base a eso, definir qué sanción aplicar."2

El encargado de subir el video de Agustín a la red social Facebook fue un compañero que intentó defenderlo y agregó en su publicación que siempre ocurren hechos violentos hacia el joven, donde lo golpean y lo dejan sin aire; y si alguien quiere defenderlo, también agreden a esa persona.
El portal de noticias La ciudad de Avellaneda, publicó el 11 de febrero de 2014 una nota sobre la preocupación ante los hechos de violencia en la vuelta a clase de los jóvenes. Sin centrarse en ningún caso específico, el texto es escrito por la Doctora Claudia Edith Fontana, directora del Centro Multidisciplinario de Resolución de Conflictos, que explica lo siguiente: "Sin duda, aquello que ocurre en la escuela es reflejo, en mayor o menor medida, de aquello que ocurre en nuestra sociedad, la que pareciera estar caracterizada por el exitismo, la competencia, los vínculos violentos, la escasez de adultos dispuestos a ocupar su rol y en la que se advierte un incremento en la desvalorización de las instituciones y de las normas." "

En septiembre del 2015, el portal postea "Promulgaron la ley que crea acuerdos de convivencia para combatir el bullying en las escuelas bonaerenses" Esta ley establece que todas las escuelas de la Provincia de Buenos Aires deberán tener acuerdos de convivencia para combatir esta problemática. "La ley prevé un abordaje integral desde la etapa inicial hasta el nivel secundario; mientras que hasta ahora ese abordaje sólo se hacía en el nivel secundario"4, destacó el legislador Marcelo Feliú, autor del proyecto. La ley promueve las campañas de concientización y una línea 0800 de asistencia.

En junio de 2017, el portal Lanús Noticias, escribió sobre un nuevo caso de Bullying en una escuela de Florencio Varela. Un chico de 16 años, cansado de las burlas por su sobrepeso, se enfrenta a su agresor dos años menor en la escuela $\mathrm{N}^{\circ} 11$ de dicha localidad. A consecuencia, el joven de 14 años sacó un cuchillo de su mochila y lo apuñaló 12 veces. Desde el portal aseguran que "La víctima fue operada, se encuentra internada y fuera de peligro en el hospital Mi Pueblo. Por otro lado, el agresor fue trasladado a la Comisaría 2da. de Florencio Varela. Allí quedó detenido a disposición de un juez de Menores." A la fecha no hay información de lo que pasó luego con ambos jóvenes. Diez días después, el portal La ciudad de Avellaneda, le dio la palabra a la Doctora Claudia Edith Fontana,

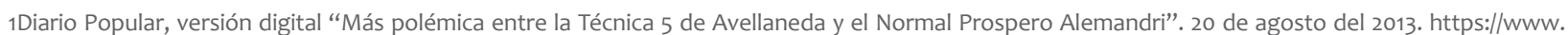
diariopopular.com.ar/sureno/mas-polemica-la-tecnica-5-avellaneda-y-del-normal-prospero-alemandri-n166655

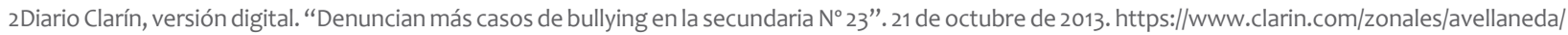
denuncian-casos-bullying-secundaria-

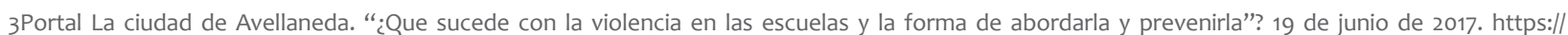
laciudadavellaneda.com.ar/que-sucede-con-la-violencia-en-las-escuelas-y-la-forma-de-abordarla-y-prevenirla/

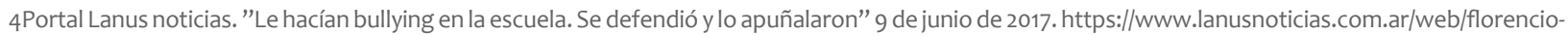
varela-le-hacian-bullying-en-la-escuela-se-defendio-y-lo-apunalaron/ 
quien menciona que "Hoy, además de la mencionada Ley Nacional 26.892 (promoción de la convivencia y el abordaje de la conflictividad social en las instituciones escolares), en la provincia de Buenos Aires contamos con la Ley 12.299 que crea el Programa Provincial de Prevención de la Violencia Escolar, y la Ley 14.750 que tiene como objeto regular la promoción, intervención e investigación de experiencias sobre convivencia y abordaje de los conflictos en las escuelas de la provincia. Es decir, que actualmente contamos con mayores y mejores herramientas que en aquel febrero del año 2014. ${ }^{6 "}$ Pese a esto, reflexionó que los casos han aumentado y cada vez se dan con mayor intensidad. Para ejemplificar, utiliza un caso de una docente agredida por una madre, extendiendo la violencia escolar a los adultos.

\section{Las voces de los/as Jóvenes ${ }^{7}$}

\section{Ana, estudiante}

Ya no es bien visto entre comillas, el hecho de burlarse de alguien, porque alguien se burlaba o era violento o malo con una persona y ahí era gracioso, entonces todos nos reíamos, pero como de a poco eso fue cambiando un montón y ya no hay tanta tolerancia como dijo mi compañero, en esa situación, entonces como que todos empezamos a tomar conciencia y una iniciativa respecto a esas situaciones.

\section{Candela, estudiante}

"Hay muchas cosas que se definen que están bien o mal, pero que no la define uno, la define la sociedad. Cuando hay una opinión de forma mayoritaria en la sociedad de que algo es bueno y de que algo es malo, es como que ya está, es como que se transmite esa idea de que ese algo es bueno y ese otro algo es malo. Entonces, fallar una vez como decías de la nena que falló en una tarea y ya se puso mal porque siente que se pudo hacerlo mejor o cualquier cosa, ya siente que va a fallar en la vida, que está todo mal.
¿Pero qué es hacer las cosas bien? Cada uno depende de las cosas que va haciendo o aprendiendo y las cosas que va viviendo, tiene una idea de lo que está bien, pero no hay que guiarse por la que te plantea la sociedad, sino que hay que plantearse uno mismo, tipo a ver, para mi ¿Qué está bien? ¿Y por qué hago esto? ¿Y con qué fin? ¿Y me va a hacer bien?"

\section{Triangulación de los resultados}

Respecto de la construcción mediática de los y las jóvenes como un enemigo, la investigadora mexicana Rossana Reguillo dice que, "mientras que en un recuadro aparece la opinión de un especialista en niños de la calle, pero esta aparece sin problematización alguna y sus opiniones, por el tratamiento que de ellas se hace, lejos de cuestionar las visiones oficiales o de confrontarlas, las confirma y el asunto se coloca en términos de comprensión para los pobres delincuentes juveniles. Se pasa así de la estigmatización al sentimiento caritativo, lo cual no permite trascender la percepción simplista que reduce la complejidad del fenómeno aludido a un asunto entre buenos y malos" (Reguillo Cruz, 1997).

Respecto del análisis estructural y conceptual podemos mencionar que, en las notas analizadas sobre casos específicos de violencia escolar, no se profundiza acerca de la definición de violencia, bullying. En ese sentido, en ninguno de los artículos se hace alusión de porque se genera dicho acontecimiento, sino que se cuenta como una crónica policial, se da detalles de cómo sucedieron los hechos y se habla de distintos tipos de violencia: Verbal, física, institucional, etc. Dicha violencia aparece expresada entre pares (alumnos), alumnos y docentes y viceversa, padres con docentes. Por su parte, en referencia a los diversos acontecimientos las notas detallan las características de la violencia siempre intentando llevar el orden establecido, de una voz de autoridad que controla la situación, una mirada normalizadora. En esa línea se valora la noticia como un hecho policial más que como una cuestión social.

5Diario Opinión Ciudadana "La coordinación de acción social brinda charlas para la erradicación del bullying y alerta sobre el grooming” 22 de Agosto de 2019 https:/www.diariopinion.com.ar/noticias/2019/08/22/27324-la-coordinacion-de-accion-social-brinda-charlas-para-la-erradicacion-del-bullying-y-alertasobre-el-grooming

6Portal Telám "Piden que se capacite a los padres sobre bullying y ciberbullying en las escuelas bonaerenses" https://www.telam.com.ar/ notas/201903/343026-piden-que-se-capacite-a-padres-sobre-bullying-y-cyberbullying-en-escuelas-bonaerenses.html

7Las voces de los/as estudiantes corresponden a diversas charlas que se organizaron en el marco del proyecto vía zoom entre septiembre del 2020 y marzo del 2021. 
Respecto a lo anterior, se reflejan en estos textos la naturalización de la "violencia escolar" que no se discute como se produce, ni la etiqueta que significa ubicar la violencia en una institución; además se plantea dicha violencia en una zona determinada, y se esboza a un joven peligroso, agresivo, con una rivalidad en ascenso con sus pares, lo que determina un pedido de autoridad y control por parte de los padres y directivos de las diversas instituciones. Por su parte en las entrevistas de los/as jóvenes de colegios secundarios queda claro que dicha problemática no puede ser analizada sin su voz. En sus expresiones se demuestra un compromiso de dialogar y reflexionar sobre dichas situaciones y fundamentalmente que son vitales en la coproducción de conocimiento, principalmente promoviendo un cambio en la perspectiva de como considerar a la violencia, diferenciándose de las situaciones que en el pasado se permitían.

Los y las jóvenes ven que en el discurso de los medios de comunicación no aparece su voz, y si ven una marcada representación en términos de sus consumos culturales y no como sujetos de derechos. En esa línea, respecto de las manifestaciones de violencia, en la representación mediática, aparecen aquellas vinculadas a agresiones físicas, pero no sólo que no explican el contexto en el cual se generan las mismas, sino también que se invisibilizan otros tipos de violencia como la simbólica, institucional, que aparecen en los discursos escolares. Desde esta mirada los medios de comunicación naturalizan la violencia, la ubican en un determinado sector de la Sociedad estigmatizando lugares y ciertos jóvenes que son marcados como peligrosos. Allí, los y las jóvenes interpelan a los medios la construcción de discursos hegemónicos y critican la presentación de discursos simplistas sin ningún tipo de profundidad, aislados de la cuestión social.

En el diálogo que se desarrolló en el marco del Proyecto y en vinculación con las lecturas de los análisis de los medios aparece la idea donde se representa la violencia como show. Esto hace que el contexto sea más violencia, y se trasladan a la escuela desde la naturalización. Cuando las consecuencias las tenemos que asumir todos. Es importante hacer una evaluación crítica de esta realidad desde la perspectiva de los/as jóvenes que hoy cuentan con espacios para la reflexión y el debate.

Los medios masivos de comunicación representan relatos, construcciones estereotipadas sin ubicar histórica, social, política. ¿Cómo construyen los medios la violencia y como se los ve a los jóvenes? ¿Qué notan de esos títulos? Son algunas de las reflexiones que dieron cuenta en las charlas en las que obtuvimos este conocimiento vital para comprender la mal llamada “violencia escolar" y promover las voces silenciadas.

\section{Conclusiones}

"Las constantes señales de la violencia son actos de crimen y terror, disturbios civiles, conflictos internacionales. Pero deberíamos aprender a distanciarnos, apartarnos del señuelo fascinante de esta violencia subjetiva, directamente visible, practicada por un agente que podemos identificar al instante. Necesitamos percibir los contornos del trasfondo que generan tales arrebatos. Distanciarnos nos permitirá identificar una violencia que sostenga nuestro esfuerzo para luchar contra ella y promover la tolerancia" (Zizek, 2009).

El proyecto generó un marco de participación de todos los actores sociales, promoviendo un espacio de prevención y concientización de las problemáticas de la mal Ilama "violencia escolar". El trabajo realizado tiene que ver con la posibilidad de democratizar la palabra y democratizar los derechos que los/as jóvenes tienen. Si uno lo analiza desde las categorías de la juventud, muchos marcos teóricos se basan en la historia de la humanidad y no consideran para el pensamiento político a los/as jóvenes. Por otro lado, hablando sobre la mal Ilamada "violencia escolar", aparece otro problema que implica atender con una única causa estos hechos. En realidad, hay causas que son múltiples, que son complejas, no sencillas como decir "el problema es que tenía una anomalía en su patología" o que escuchaba Marilyn Manson, o que había visto dos días atrás el documental Bowling for Columbine de Michael Moore, o que el problema es la familia.

Uno de los datos relevantes en estos análisis respecto a esta problemática, es que generalmente los hechos para los medios o para la comunicación se cuentan de forma de lo que es la vieja historia crónica policial, que te dice "Junior entró al aula, dio tantos pasos, hizo esto, hizo lo otro" y bueno, el caso queda ahí. En el contexto de lo que se denominó la Masacre de Carmen de Patagones una de las cosas que publicaron los medios fue la frase que escribió Junior en su pupitre y que decía "si alguien le encontró un sentido a la vida, por favor escríbalo aquí". 
¿Porque para un joven de 16-17 años no tiene sentido ni su vida o la de los demás? ¿Qué le hemos dado, qué le hemos ofrecido como sociedad como modelo para que a esa corta edad diga que no tiene sentido la vida? Para tratar de comprender está interrogantes debemos preguntarnos que damos como naturalizadas y que permiten ver como se cataloga o etiquetan estos hechos: ¿Qué se señala como violencia en las escuelas? ¿Qué sentido construyen los medios de comunicación sobre la violencia y las causas de ella? ¿Cómo opera la escuela frente a los hechos de violencia? Estas preguntas nos permiten reconstruir los diferentes sentidos que adquiere este concepto en el imaginario social y de qué modo interfieren los medios de comunicación en esa construcción.

Pensar la posibilidad y conveniencia de utilizar la tecnología audiovisual con carácter educativo, para prevenir estos fenómenos. De este modo, el relevamiento previsto apuntó a ver de qué modo los medios construyeron la realidad sobre la violencia escolar, bajo qué parámetros definieron estos sucesos y cómo identificaban a los jóvenes. En definitiva, nuestro desafío es escuchar y comprender a los/as jóvenes solo así podremos prevenir esta problemática.

\section{Referencias bibliográficas}

- Barbero, Jesús Martín (1989). Violencias televisadas. Ponencia presentada en la I Conferencia de Facultades de Comunicación y Periodismo, convocada por la Unión de Universidades de América Latina, (UDUAL), realizada en la Universidad Central de Bogotá del 13 al 16 de septiembre de 1988, y publicada en la Rev. "Hojas universitarias” Vol. IV, No. 33, Bogotá.

- Barreras, Luis (2012). Jóvenes, violencia y medios. En la revista Novedades Educativas $N^{\circ} 254$, Pág 1317. Argentina-México.

- Margulis, Mario (1996). La juventud es más que una palabra. Ensayos sobre Cultura y Juventud. Ed. Biblos. Buenos Aires.

- Medina, Juan Manuel; Tommasino, Humberto (2018). "Extensión Crítica: Construcción de una Universidad en Contexto". Rosario-Argentina: UNR.

- Reguillo Cruz, Rossana (1997). Crónica roja: espectáculo y negocio. Revista Chasqui № 60.
- Reguillo Cruz, Rossana (2000). Emergencia de culturas juveniles. Estrategias del desencanto juvenil; en Enciclopedia Latinoamericana de Sociocultura y Comunicación. Buenos Aires, Ed. Norma.

- Reguillo Cruz, Rossana (2005). Revista Tram(p)as de la Comunicación y la Cultura. Pág. 6. Año 4.

- Sautu, Ruth (2003). Todo es teoría: objetivos y métodos de investigación. - la ed. - Buenos Aires : Lumiere.

- Wainerman, C. y Sautu, R. (1997). La trastienda de la investigación, Buenos Aires, Editorial de Belgrano.

- Zizek, Slavoj (2009). Sobre la violencia. Seis reflexiones marginals. Ed. Paidós. Bs.As 
COMPROMISO SOCIAL. Revista de la UNAN-Managua, Extensión Universitaria, $\mathrm{N}^{\circ}$ 5, Año 03. Vol 1 Ene-Jun. 2021. 